\title{
Scénario catastrophe : désordre après la guerre froide. Partie 2
}

John Mueller

\section{(2) OpenEdition \\ Journals}

Édition électronique

URL : http://journals.openedition.org/conflits/880

DOI : $10.4000 /$ conflits.880

ISSN : $1777-5345$

Éditeur :

CCLS - Centre d'études sur les conflits lilberté et sécurité, L'Harmattan

Édition imprimée

Date de publication : 15 octobre 1995

ISSN : 1157-996X

\section{Référence électronique}

John Mueller, «Scénario catastrophe : désordre après la guerre froide. Partie 2 », Cultures \& Conflits [En ligne], 19-20 | automne-hiver 1995, mis en ligne le 01 mars 2003, consulté le 30 mars 2021. URL : http://journals.openedition.org/conflits/880 ; DOI : https://doi.org/10.4000/conflits.880

Ce document a été généré automatiquement le 30 mars 2021.

Creative Commons License 


\title{
Scénario catastrophe : désordre après la guerre froide. Partie 2
}

\author{
John Mueller
}

Changements de définitions

1 Dans le sillage de la guerre froide, pour susciter et justifier l'inquiétude, les "identificateurs de troubles" ont ingénieusement changé le sens de certains mots-clé. L'un d'eux est "stabilité". Durant la Guerre Froide, l'instabilité était clairement associée au risque de guerre nucléaire entre les Etats Unis et l'Union Soviétique. Il n'est peutêtre pas inutile de rappeler à ce propos qu'il y a encore quelques années, bien des gens étaient profondément inquiets de voir éclater une telle guerre. Vous vous souvenez de l'épée de Damoclès? Des deux scorpions dans la bouteille? De l'horloge du Jugement Dernier sur la couverture du "Bulletin of Atomic Scientists" ? En 1945, le diplomate américain Joseph Grew, réputé pour sa clairvoyance, concluait : "la probabilité d"une guerre avec l'Union Soviétique est aussi certaine que n'importe quoi d'autres dans ce monde"1. En 1950, Arnbold J. Toynbee écrivait: "Au cours de l'histoire récente de l'Occident, les guerres se sont succédées avec de plus en plus d'intensité et de rapidité et il semblerait aujourd'hui que la Guerre de 39-45 ne soit pas le point culminant de ce mouvement en crescendo". Parallèlement, Albert Einstein était persuadé qu'"à moins que nous ne soyons capables dans un avenir proche de supprimer la crainte mutuelle d'une agression militaire, nous sommes condamnés". En 1969, le stratège et futurologue Herman Kahn estimait pour sa part: "j'ai la ferme conviction que si nous ne réfléchissons pas sérieusement sur les différentes implications du problème stratégique, nous n'atteindrons pas l'an 2000, et peut-être même pas l'année 1965, sans qu'il ne se produise un cataclysme". C. P. Snow confirmait devant ses auditeurs qu'"à moins de limiter sévèrement les armes nucléaires, il est "certain" qu'au cours des dix prochaines années, au plus tard, certaines de ces bombes nucléaires auront explosées. Le réaliste Hans J. Morgenthau concluait en 1979 que "le monde se dirige de manière inéluctable vers une Troisième Guerre Mondiale - une guerre nuclèaire stratégique. Je ne crois pas que l'on puisse faire quoique ce soit pour l'empêcher. Le système international est tout simplement trop instable pour survivre plus longtemps". En 1982, Jonathan Schell proclamait dans un best-seller que "un jour - et il est difficile de croire 
que ce ne sera pas bientôt- nous devrons choisir. Soit nous sombrerons dans le coma terminal et tout prendra fin, soit, comme je le pense et j'ai confiance en cette idée, nous prendrons conscience du péril que nous courrons (...) et nous nous dresserons pour débarrasser la terre des armes nucléaires". Récemment encore en 1987, des sondages révélaient qu'un quart de la population américaine considérait la menace de guerre comme l'un des problèmes les plus importants auxquel était confronté le pays" ${ }^{2}$.

Il reste encore beaucoup d' armes nucléaire sur terre, mais avec la disparition de la Guerre Froide, il est clair que, comme le note Gates, "le danger d'une guerre majeure en Europe ou d'une guerre thermonucléaire globale s'est estompé au point de disparaitre". Ainsi, si l'on considère que la stabilité est renforcée à chaque fois que les grandes puissances nucléaires s'éloignent un peu plus d'un conflit potentiel entre elles, alors aujourd'hui le monde baigne certainement dans la stabilité.

3 Pour les oiseaux de mauvais augure, il existe deux solutions au dilemme posé par cette évolution pourtant souhaitable. La première est de nier allègrement que la menace d'une guerre thermonucléaire était si grande. Ainsi, Karen Elliott House dans le Wall Street Journal concluait calmement que "le monde de l'après-Guerre Froide est effectivement moins menaçant si on l'on part du point de vue simpliste selon lequel la confrontation des superpuissances appartient pour le moment au passé". En suivant son raisonnement, des décennies de traumatismes et de peurs n'ont pas véritablement existé.La seconde solution possible est de modifier prestement la définition de la stabilité en arguant que les affrontements sanglants et les conflits frontaliers doivent être considérés aujourd'hui comme des signes d'instabilité. Maispour être cohérent, il faut reconnaître, comme je l'ai fait auparavant, que la Guerre Froide était selon ce critère très instablepuisque des affrontements sanglants et des conflits frontaliers ont eu lieu partout et dans la mesure où le conflit entre Grandes Puissances était dans la plupart des cas plausibles et le plus souvent bien réel.

D'autres termes se sont vus attribuer de nouvelles définitions: par exemple "guerre majeure" et "conflit global". Avant 1989, les guerres majeures ou les conflits globaux désignaient des batailles dans lesquelles les grandes nations étaient profondément et directement engagées: le type d'événement qui a caractérisé la Première et la Deuxième Guerre Mondiale. Cependant, l'ancien Président des Etats-Unis Jimmy Carter, dans un discours portant sur la politique étrangère à la Convention Démocratique Nationale en 1992, (un évènement remarquable dans ce genre de réunion), annoncait que se déroulaient 35 "guerres majeures" actuellement dans le monde. Sous le terme de "guerre majeure", il désignait tout conflit au cours duquel au moins 1000 personnes avaient été tuées ${ }^{3}$. Il avait ainsi retenu une définition classique de la guerre mais l'avait rebaptisée "guerre majeure"4. Apparemment pour Carter les guerres sont comme les olives californiennes : elles sont toutes au minimum gigantesques. De même, dans une recension dans le New York Times concernant le livre de Michael Howard, publié avant la Guerre du Golfe, Herbert Mitgang qualifiait de prophétique l'observation de M. Howard qui estimait "s'il y a un endroit dans le monde aujourd'hui qui pourrait être à l'origine d'un conflit global c'est le Golfe Persique". Cette affirmation peut être effectivement considérée comme une prophétie si on élève la Guerre du Golfe au rang de conflit global. Mitgang ajoute plutôt de manière confuse qu'"après deux Guerres Mondiales, il est difficile de faire la distinction entre guerres locales et guerre de grande échelle". On aurait pu croire que la distinction restait somme toute aisée, après tout les différences ne sont pas minces. Un autre tour de passe-passe verbal semble 
avoir été utilisé pour ce que Woolsey appelle "les armes de destruction massive". A une certaine époque cette expression était réservée aux seules armes nucléaires mais petit à petit elle a été élargie aux armes chimiques - procédés beaucoup moins efficaces lorsqu'il s'agit de tuer en masse.

Cet exercice de redéfinition permet en outre aux oiseaux de mauvais augure professionnels de résoudre une difficulté supplémentaite liée à la prolifération nucléaire "alarmante" de ces dernières années. Le National Planning Association anticipait "une augmentation rapide du nombre de puissances atomiques ... pour le milieu des années 60". En 1960, Snow prévoyait que "dans les six prochaines années la Chine et plusieurs autres Etats détiendraient un arsenal de bombes nucléaires". Comme le soulignait Stephen Meyer, dès lors qu'ils maitrisent la capacité technique, il n'y a pas nécessairement urgence à ce que ces pays deviennent des puissance nucléaires. Denis Healey, à cette époque Ministre britannique de la Défense, notait pourtant que "jusqu'ici, aucun pays n'a résisté à la tentation de fabriquer ses propres armes atomiques une fois acquise la technologie pour les construire". Même à cette époque, ce n'était pas exact. Le Canada aurait pu se doter d'armes nucléaires s'il l'avait souhaité et c'est plutôt cette voie canadienne qui semble avoir été la plus fréquemment choisie. En fait, une des plus intéressantes évolutions dans le monde de l'après-guerre est clairement la lenteur du processus de prolifération des armes nucléaires. Par ailleurs, de nombreux pays comme le Brésil, l'Argentine, la Corée du Sud, Taiwan, ont suspendu ou renoncé à leurs programmes nucléaires. Sans doute l'hostilité des puissances nucléaires y a joué un rôle non négligeable. Mais cela semble dû aussi au fait que bon nombre de pays potentiellement nucléaires ont renoncé à accorder quelqu'intérêt à ces armes coûteuses. Le Japon et l'Allemagne jouissent d'un grand statut dans le monde bien qu'ils ne soient pas détenteurs d'armes nucléaires. Accorderait-on plus de crédit à la Grande Bretagne ou à la France si ces derniers possédaient une batterie de 50000 armes nucléaires, leur accorderait-on moins d'attention s'ils n'en avaient aucune? Les armes nucléaires détenues par Israël n'ont pas dissuader les Arabes de l'attaquer en 1973 et celles de la Grande Bretagne n'ont pas permis d'empêcher l'invasion des Falklands par l'Argentine en 1982. Et des dizaines de milliers d'armes nucléaires aux mains des forces alliées n'ont pas convaincu Saddam Hussein de retirer ses forces du Koweit en 1990. Ainsi, la prolifération nucléaire a été désepérement lente si l'on se place du point de vue des plus pessimistes. Certes, si on élargit le champ de la prolifération en y incluant d'autres armes et en affirmant qu'elles sont identiques aux armes nucléaires, alors on trouvera forcément toujours matière à s'inquiéter. Woolsey et bien d'autres peuvent bien s'inquièter tout spécialement de la prolifération des missiles balistiques, mais comme l'a souligné Thomas Mac Naugher, cette préoccupation est déplacée, les missiles sont en effet des armes coûteuses, peu fiables et elles ne rivalisent pas avec la force aérienne dès lors qu'il s'agit de délivrer des armes chimiques et conventionnelles ${ }^{5}$. Bien sûr, il peut être intelligent d'encourager ces pays à gaspiller leur argent dans ce type de système d'armes plutôt que de les voir acheter des avions moins coûteux et beaucoup plus efficaces.

Des critères toujours plus exigeants

6 La légende qui accompagnait l'exposition d'un vieil aspirateur au Musée strong de Rochester à New York illustre bien ce phénomène de réévaluation des critères d'appréciation. On pouvait y lire: "Les inventions techniques qui ont permis d'économiser de la main d'œuvre telles que cet aspirateur ont aidé les classes moyennes à satisfaire leur désir de propreté domestique". Cet aspirateur représentait 
très certainement un réel progrès cependant la légende poursuivait : "Chaque nouvelle technique porte en elle de nouvelles exigences et l'idéal de la propreté reste toujours aussi difficile à atteindre". Ainsi, rien ne va jamais mieux! Stanley Hoffman nous suggère par exemple qu'"une politique d'ordre mondial nécessiterait que les nombreuses sources de troubles mondiaux ou régionaux soient abordées d'une manière telle qu'elle puisse minimiser les conflits violents entre pays, réduire l'injustice entre et à l'intérieur des pays et empêcher les violations graves des droits fondamentaux en leur sein"6. Mais comme le notent Max Singer et Aaron Wildavsky, Hoffmann s'appuie sur une conception extrêmement restrictive de l'ordre selon "des critères jamais atteints dans l'histoire de l'humanité".Cette conception aboutit inévitablement à la conclusion logique selon laquelle les obstacles à une telle politique demeurent énormes", ce qui est effectivement un doux euphémisme ${ }^{7}$. De même, Gates défend le point de vue selon lequel nous vivons aujourd'hui dans un monde "où l'instabilité, les turbulences et la violence se sont généralisées et dans lequel personne ne peut plus prévoir le cours des événements". Cela signifie que le critère de l'ordre choisi est proche de la perfection, puisque pour l'atteindre il faut dans ce cas que l'instabilité, les turbulences et la violence soient réduites à néant et la prédiction parfaite. Parallèlement, Zbigniew Brzezinski publia un livre alarmiste dans lequel il affirmait que "les bouleversements du monde ne sont plus maîtrisés", avouant implicitement qu'il fut un temps où ils étaient bien contrôlés. Huntington quant à lui estime que "quelque soit le côté vers lequel on se tourne, il n' y a que des désaccords", formulation suffisamment vague pour avoir toujours été et qui sera toujours vraie. Le Ministre des Affaires Etrangères norvégien Johan Jorgen Holst observait que "la notion d'un danger clairement identifié et actuel a été remplacée par un ensemble vague de risques et de dangers"8. Mais les conflits, les troubles, sans parler des risques et des dangers diffus sont inévitables parce qu'il est impossible que tout le monde défende les mêmes intérêts. Souhaiter leur éradication est parfaitement absurde. Les commentateurs appliquent régulièrement ce type de raisonnement dans leurs analyses sur les pays récemment devenus démocratiques. Ils y déplorent la corruption qui a accompagné le développement $\mathrm{du}$ capitalisme dans les anciens pays communistes, oubliant allègrement que le système communiste était totalement corrompu, que la corruption est un phénomène florisant dans des pays développés comme le Japon. Ou alors ils se plaignent de l'incapacité de certains pays fraîchement démocratiques à remettre de l'ordre, tout en passant sous silence le fait que dans la campagne présidentielle américaine on n'a pas arrêté de se plaindre et de se lamenter sur les blocages institutionnels qui font pourtant l'essence même de la démocratie américaine.

Exagérer l'importance des problèmes mineurs.

7 Finalement, lorsque les problèmes majeurs (appelés « dragons ») dans la taxinomie de Woolsey) disparaissent, les problèmes mineurs («serpents ») peuvent se voir accorder une plus grande importance. Woolsey a identifié d'une manière qui l'arrange les serpents qui sèment le désordre dans notre nouveau monde : "la prolifération d'armes de destruction massive et les missiles balistiques qui les transportent, les haines ethniques et nationalistes susceptibles de se propager à travers le monde, le trafic international de drogue, le terrorisme, les dangers qu'impliquent la dépendance de l'Ouest à l'égard du pétrole du Moyen-Orient et enfin les nouveaux défis économiques et environnementaux. » Mais il se trouve qu'aucun des serpents venimeux de Woolsey n'est nouveau et que certains d'entre eux sont en réalité un sujet de préoccupation moins urgent qu'ils ne l'étaient durant la Guerre Froide. 
Comme nous l'avons dit plus haut, les guerres issues des haines ethniques et nationales ne sont ni un phénomène récent ni plus fréquent qu'auparavant. La prolifération nucléaire n'est plus non plus un problème nouveau - en fait il se pourrait même qu' elle représente une difficulté bien moins épineuse qu'en 1960 lorsque John Kennedy ne cessait de faire répeter avec inquiétude qu'il se pourrait que 10,15 ou 20 nations atteignent la capacité nucléaire d'ici à 1964 (Kraus1962, 394). Le trafic de la drogue international sévit aussi depuis un certain temps alors que la dépendance des pays de l'Ouest à l'égard du pétrole moyen-oriental a été un sujet de préoccupation reconnu depuis au moins 1973.

9 L'impact du terrorisme international résidait davantage dans l'hystérie exagérée qu'il générait que dans ses effets physiques réels. Il y a eu moins d'américains tués par des terroristes que par la foudre au cours de la dernière décennie. Ainsi, malgré une recrudescence en 1991 à l'époque de la Guerre du Golfe, le terrorisme s'est constamment réduit à partir des derniers jours de la Guerre Froide, notamment grâce au renforcement des mesures de prévention et à un meilleur travail de police internationale. Peu de personnes veullent se souvenir de la fréquence et de la mode des détournements d'avions et encore moins nombreux sont ceux qui se souviennent de la très vive inquiétude suscitée durant la Guerre Froide par les Brigades Rouges en Italie, par la bande Baader-Meinhoff en Allemagne, par les Gardes Rouges au Japon et par le Front de Libération Symbionaise aux Etats-Unis'. Cependant, malgré tout cela, Gates prévoit avec assurance que « la courbe du terrorisme va croitre de manière constante. » Les défis économiques et environnementaux ne sont guère récents non plus mais on sonne malgré tout le toccin.Certains ont cru décelé un dangereux ennemi là-bas sur le front économique: le Japon insidieusement pacifique. Ceux qui se réclament de l'école «Alerte! Le Japon achète Pearl Harbour $! »^{10}$ comme Huntington affirment que nous ne devons plus craindre la «vulnérabilité des missiles» mais "la vulnérabilité semiconductrice ». Il nous prévient le plus sérieusement du monde que « l'économie est la continuation de la guerre par d'autres moyens ». Il affirme ainsi que la question est aujourd'hui de savoir si les Etats-Unis seront capables de relever le défi économique lancé par le Japon avec autant de succès qu'ils ne l'ont fait sur un plan politique et militaire avec l'Union Soviétique ${ }^{11}$. Si ce n'est pas le cas, les Etats-Unis perdront irrémédiablement leur suprématie dans une arène de pouvoir cruciale ». Selon lui, il faut tirer la sonnette d'alarme parceque le Japon est devenu le plus grand fournisseur d'aide étrangère et parcequ'il a été jusqu'à subventionner des chaires à Harvard et au Massachussets Institute of Technology ${ }^{12}$.

11 Vers le milieu de l'année 1993, l'économie japonaise a cependant sombré dans la dépression tandis que l'économie américaine commenÁait à bien se porter. Huntington, toujours le plus souple et le plus inventif des oiseaux de mauvais augure se mit alors à faire des extrapolations à partir de la guerre civile en Bosnie et proclama qu' en fait « la cause fondamentale des conflit» ne sera donc pas principalement »idéologique ou économique mais plutôt, que les grandes divisions de l'humanité et la principale source de leur conflit seront culturelles $»^{13}$. Il y aurait environ sept ou huit civilisations principales dans le monde et ces civilisations sont destinées à s'affronter globalement surtout à leurs confins $»^{14}$. Que la civilisation occidentale ait soutenu à l'origine la création d'un état en Bosnie qui sera dominé par la civilisation islamique trouble si peu Huntington qu'il ignore la question ${ }^{15}$, occupé qu'il est à nous convaincre que les idéaux occidentaux de « libéralisme, de constitutionnalisme, de Droits de l'Homme, d'égalité, 
de liberté, d'état de droit, de démocratie, d'économie de marchés » trouvent peu d'écho dans des endroits tels que le Japon qui a, que je sache, un état de droit, libéral, scrupuleux des lois, démocratique, constitutionnel et capitaliste ${ }^{16}$.

Il existe encore d'autres problèmes. Dans son best-seller pessimiste de 1993 « Préparer le Vingt-et-unième Siècle ", l'historien Paul Kennedy s'ingénie à nous inquiéter par défaut sur la pollution, l'immigration et la robotique. Il est assez intéressant de noter que la guerre, préoccupation centrale de son best-seller de 1987, a apparemment disparu du champ de ses préoccupations : le mot «guerre » n'apparait même pas dans l'index de son dernier livre.

Bien entendu, il est toujours possible de trouver des sujets de préoccupation nationale. Brzezinski voit des troubles partout et rejette pêle mêle la responsabilité sur la richesse matérielle, sur le laisser-aller et sur l'éternel blitzkrieg mediatique de la télévision. Autre possibilité ; nous pouvons redécouvrir les problèmes de haine raciale comme s'ils n'avaient jamais existé. Nous pouvons aussi geindre sur les problèmes économiques liés à l'allongement de la durée de vie et l'amélioration des soins médicaux, ou alors nous ronger les sangs sur la question de savoir s'il est devenu nécessaire d'augmenter les taxes sur l'essence ou s'il faut revoir la réglementation de la télévision par cable ${ }^{17}$.

On pourra même déplore le fait que "les américains sont dépassés, voire paralysés " par tous les choix auxquels ils sont confrontés sur le marché. Ainsi, David Goslin nous affirme que « en tant que sociologue, nous savons que plus il y a de choix, plus les gens tendent à devenir anxieux "; Todd Gitlin fait observer que "si vous vous trouvez face à une infinité de choix, les gens sont réduits à la passivité » et le futurologue Alvin Toffler s'inquiète du « surchoix - o $\mathrm{o}^{\smile}$ les avantages de la diversité et de l'individualisation sont annulées par la complexité du processus de décision de l'acheteur ${ }^{18}$. Il est clair en effet que si Hamlet faisait face à une seule alternative et qu'il s'en trouvait torturé pendant les cinq actes de la pièce, alors effectivement nous pouvons considérer que notre situation est vraiment pire aujourd'hui. Cette anecdote semble être une version revisitée du casse tête aristotélicien classique connu sous le nom de l" »âne de Burridan » dans lequel l'animal est placé à égale distance de deux bottes de foin et meurt finalement de faim à cause de son interminable indécision. Il semble n'y avoir aucune preuve de l'existence d'un âne ayant subit une telle agonie mais plus sérieusement ce type de problème devrait d'urgence nécessiter un programme complet de recherche ${ }^{19}$.

15 Ainsi, misanthropes et vieux grincheux peuvent se féliciter mutuellement. Même dans un état de paix notoire, il y aura toujours matière à se plaindre et à s'inquiéter : le scénario catastrophe sera toujours le plus facile à écrire. Puisque l'éventualité d'une catastrophe thermonucléaire globale (sans doute une guerre tellement dingue sur l'échelle de Carter) a diminué à tel point que plus personne ne s'en soucie, il y aura toujours quelqu'un pour attirer notre attention sur des ennemis plus nébuleux: le trouble, le chaos, l'incertitude, l'imprévisible, l'instabilité ou les risques et les dangers diffus. Ce genre d'ennemis - comme l'économie, la civilisation et le choix- seront assurément toujours avec nous.

Michael Howard fait observer que "chaque nouvelle génération se présente avec de nouveaux problèmes et des nouveaux défis $»^{20}$. C'est certainement vrai mais pourrions nous aussi faire une petite pause pour fêter l'éradication de la variole ou le déclin de la menace d'une guerre thermonucléaire globale et suggérer qu'un monde débarrassé de ces fléaux est tout compte fait meilleur.Au bout du compte, il apparait clairement que si 
jamais nous entrons un jour dans l'âge d'Or dont parlait Truman, on ne le remarquera jamais. "Status quo » comme l'aurait dit Ronald Reagan, est l'expression latine pour «le désordre dans lequel nous nous trouvons. »

\section{NOTES}

1. . cité par Gaddis, op. cit., 218n.

2. . Mueller J., Policy and opinion in the Gulf War, Chicago, University of Chicago Press, 1994, registre 45.

3. . MacNeil / Lehrer NewsHour, 15 février 1993.

4. . Singer J. D., Small M., The wages of war. 1816-1965 : a Statistical handbook, New York, Wiley, 1972, p. 49.

5. . Mac Naugher T. L., " Ballistic missile and chemical weapons : The legacy of the IranIraq war », International Security, 15, fall, 1990, p. 32-33.

6. . Hoffmann S., op. cit., p. 38.

7. . Hoffmann S., op. cit., p. 191.

8. . Holst J. J., « European and Atlantic security in a period of ambiguity », The World Today, December, 218-21.

9. . Sur le travail couronné de succès des polices espagnoles et françaises contre les terroristes basques, voir le New York Times, 11 mars 1993, A 5.

10. . Le concept de "guerre économique » en arrive presque à être oxymoronique. $\mathrm{Ce}$ concept pourrait parfois avoir du sens (comme lorsque le monde s'est associé contre l'Irak en 1991), mais la guerre représente en substance une somme égale à zéro (ou négative), tandis que l'échange économique, bien que pas nécessairement juste ou équilibré, donne généralement une somme positive par le gain des deux parties. Voir Jervis R., ' "International primacy : Is the worth the candle? ", International security, 17, spring, 1993, p. 52-67.

11. . Samuel Huntington, op. cit., p. 16.

12. . Huntington S. P., « Why international primacy matters », International security, 17, spring, p. 77-80.

13. . Huntington S. P., « The Clash of Civilization », Foreign Affairs, $n^{\circ} 72$, summer, 1993 , p. 22.

14. . Op. cit., p. 25. Huntington affirme d'autre part que « les guerres se produisent les plus fréquemment entre sociétés avec de hauts niveaux d'intéraction » (« if not civilizations, then what ?..op. cit., p. 192., Des ruptures aussi violentes aussi violentes sont probablement encore plus susceptibles de se produire à l'intérieur des civilisations.

15. . Op. cit., p. 37-38.

16. . Huntington défie ses (nombreux) détracteurs de proposer « une meilleure explication de ce qui passe dans le monde » (« If not civilization... », op. cit, p. 194). L'observation de Thomas Friedman s'impose d'elle même. Selon lui le monde est en train de se diviser deux avec d'un côté les Etats enthousiastes, tels que le Japon, qui 
fabrique sans efforts de superbes produits comme l'automobile Lexus, et de l'autre les Etats réticents, comme la Serbie qui se dispute sur la question de savoir à qui appartient tel cerisier. Bien que les constructeurs de Lexus dans le monde soit désireux de consacrer de l'argent et un petit nombre de vies pour aider les combattants des cerisiers à résoudre leurs disputes, ils sont surtout déterminer, face à l'échec d'une résolution conhérente de ces conflits, à contenir et isoler ces derniers tandis qu'ils continuent de poursuivre leur objectif premier : s'enrichir toujours plus. Voir également Rosecrance R., The rise of the trading state : Conquest and commerce in the modern world, New York, Basic Books, 1986.

17. . En 1993, il a été annoncé que l'espérance de vie, à la naissance, des américains, avait augmenté pour atteindre le chiffre record de 75 ans $1 / 2$. Cette information était si peu importante que le New York Times avait simplement reproduit un article de l'Associated Press sur ce sujet et l'avait enterré à la treizième page de son numéro du 1er septembre.

18. . Cité par Williams L., "Free Choice : When too much is too much », New York Times, 14 February, 1990.

19. . Cependant, le problème peut se résoudre de lui même dans une zone. Si les clients des supermarchés étaient paralysés par une indécision anxieuse devant des Corn Flakes, par exemple, ils bloqueraient les allées. Cela réduirait les bénéfices du propriétaire du magasin qui serait alors logiquement forcé d'agrandir les allées ce qui en retour réduirait l'anxiété du choix auquel était confronté auparavant le client.

20. . Op. cit., p. 5.

\section{INDEX}

Mots-clés : (in)sécurité, menace, prévention et préemption, Relations Internationales, risques Index chronologique : post-guerre froide 